\title{
Discussion on the Relationship between Urban Planning and Design and Architectural Design
}

\author{
Weifang Binhai Construction Project Working Drawings Review Co.,Ltd, Shandong, Weifang ,261041
}

\section{The relationship betw-een architectural design and urban planning and design Introduction}

1.1 Architectural design is the basis of urban planning and design

(1) Architectural design should embody humanistic style. Architecture not only provides the needs for people's daily life, but also is an important bearing of national culture and local customs. Therefore, in the process of architectural design, in order to fully reflect the humanistic style, it is necessary to combine the city's historical background, the development process with the needs of people, so that the humanistic care, people-oriented and humanistic spirit and other ideas can be fully demonstrated. For architectural designers, they should adapt to the needs of the times development and rationally apply artistic techniques. Therefore, the building can be endowed with emotion and the city people's living status as well as the architectural culture and taste can be embodied.

(2) Architectural design should demonstrate the function of the city. Urban planning and design and architectural design provide reasonable layout and planning for people's daily necessities, so as to build a beautiful city blueprint. Architecture is the best embodiment of urban function, and it is also the most basic factor to realize the city function. In the process of urban architectural design, the spatial division of industrial area, residential area and business district is clear and design standards of buildings are different in different areas. For example, due to the larger flow of people, shopping malls in the business district should have strong fire fighting function. In the process of school building design, it should be ensured that it has good anti-seismic function so as to avoid endangering students ' safety when the earthquake occurs ${ }^{1}$.

(3) Architectural design should embody the main landscape of the city. Buildings display the city's theme and style. The distinctive and unique architecture is an important symbol and standard of city, which is not only represent the city's history and present, but also demonstrates the goal of the future development direction of the city. So the buildings can also be called the main landscape of the city. Distinctive buildings should be created in accordance with the city planning, bringing people refreshing feeling and new development vitality.

\subsection{Architectural design should conform to urban planning}

In the process of urban planning and design, the future development and layout of the city as a whole is an important prerequisite. It is necessary to extend the history of the city and to promote the development of urban society, life and economy. Architecture

\begin{abstract}
The urban planning and design is closely connected with the architecture design. In view of the development of the whole city, only by combining architectural design with urban planning and design closely and effectively can we promote the harmonious and steady development of the city.Therefore, it is necessary to deal with the relationship between the two scientifically, so as to combine the architectural design and the urban planning organically to promote the common progress.Based on this, the paper analyzes the relationship between urban design and architecture design, hoping to provide an effective reference for better improving the level of urban design and architecture design.
\end{abstract}

Key words: Urban design ; Architectural design ; Relationship

Published online: 30th Sept, 2017

is an important foundation and an essential part of the city. It is also an important display of urban characteristics. In the process of architectural design, the building should also be 
adapted to the urban planning and design. At the same time, considering the urban environment as an important factor, the architecture should be fully integrated into the environment so as to create a suitable place for people's leisure and life.

2. The effective path to correctly deal with the relationship between urban planning and architectural design.

\subsection{Scientific design for urban planning}

Urban planning is an important content of social practice, which can promote wider development of society and material space $^{2}$. In the process of actual urban planning and design, its content should be gradually developed from a pure form to a comprehensive design and a variety of requirements should be considered comprehen-sively, such as the color of materials, quality and shape, etc. The urban planning should be scientifically analyzed and it can be combined with the society, so that the daily life needs of residents can be ensured to better promote the development of urbanization process. In recent years, the content of urban planning and design has been integrated gradually, and the techniques and methods adopted are more and more diversified.

2.2 Architectural design relies on urban palnning

At present, with the increasing development of our country's economy, the problems in architectural design are becoming more and more prominent. Some designers do not fully consider the surrounding environment and blindly pursue uniqueness in the process of designing the building monomers or groups. For example, applying the foreign architecture design concept, the decoration of the Chinese baroque building in Harbin is very gorgeous. However, it is because of the ornate decoration that the structure of the building itself is not reflected, thus losing the cultural deposits. Similarly, the same is true of the design of Chang'an Avenue in Beijing. Although the design of decoration is relatively reasonable, it is only simple to see the artistic value of architecture and it is so incompatible in the process of integration with the surrounding environment, so that its aesthetic greatly discounted. Therefore, in the actual architectural design process, the city planning should be the important basis to carry on the reasonable design, so that the construction and the environment can be fully integrated.

2.3 Strictly control design quality

In order to ensure the design quality, it is necessary to ensure the rationality of the design plan. The construction data of the project is required to be comprehensive, and the field investigation work should be done so as to make a reasonable plan in line with the urban construction ${ }^{3}$. First, the design outline should be formulated by professional technical staff and then reviewed by the relevant departments. After the audit, the program can only be implemented. In the process of implementation, it should be based on relevant standards. After the completion of the construction, the acceptance shall be completed. If there is a defect problem, it should be improved in a timely manner. 2.4 Establish a review system of architectural design scheme

Architecture is an important symbol of the city and its design no matter good or bad has an important relationship with the city image. Therefore, it is necessary to establish a sound evaluation system for architectural design scheme, and organize some experts to make reasonable assessment of the design scheme: first of all, whether the construction plan is in conformity with the requirements of urban planning and design; Secondly, the architectural design scheme is reviewed to examine whether the design idea is consistent with the requirements of the design mission. In this way, we can improve the level of architectural design and promote urban development.

In a word, architecture is the foundation of urban construction and is the most important part of the city, which is related to the style and layout of the city as a whole. Urban planning is the basis of urban construction, and there is an inseparable connection between the two. Therefore, it is necessary to continuously improve the architectural design, so that the market competitiveness of enterprises can be greatly improved. The relationship between architectural design and urban planning should be properly handled, which will be helpful to build a more harmonious and beautiful city.

\section{References}

[1] Li Wei.Analysis on the Relationship between Urban Planning and Design and Architectural Design [J]. Residence and real estate, 2017,09:123.

[2] E Haijun.Analysis on the Coordinated Development of Urban Planning and Design and Architectural Design [J]. Residence and real estate, 2017,09:102.

[3] Zhang Jing.On the Relationship between Architectural Design and Modern City Characteristics [J]. Intelligent city, 2017,03:195. 